\title{
Development and Implementation of the College Students' Psychological Consultation System
}

\author{
Kun Wang ${ }^{1, a}$, Guang Cheng Cui ${ }^{1, b}$ and Lin $\mathrm{Li}^{1, \mathrm{c}}$ \\ ${ }^{1}$ Qiqihaer Medical University, Qiqihaer, 161006, China \\ aemail: wangkun2789@126.com, bemail: gccui@163.com, cemail: 9117071@qq.com
}

Keywords: Psychological consultation system; Psychological test; ASP.NET; SQLServer; Web

\begin{abstract}
With an increase of social competitive pressure and a change of people's working and life styles, the psychological problem has become an increasingly severe common issue in the current society. Due to specificity of psychological consultation, and because traditional consultation modes are constrained by various factors on looking into psychological diseases, protecting personal privacy and improving service efficiency, hence, most current consultation systems exist problems like bad system real-time performance and inclusiveness, weak data analyzing function and worse security. Aiming at above problems, the paper studies and develops a Web-based psychological consultation system. And this system in the paper is characterized by advantages such as low cost, convenient accessing and real-time interaction, realizes an all-to-all online interactive consultation service that users are not limited by time and region, and meets many kinds of consultation requirements by vast psychological consultants, possessing highly application values.
\end{abstract}

\section{Introduction}

In recent years, the psychological problem of college students is increasingly prominent and also presents a trend of rising development. It occurs in everybody, and if it cannot be solved, obstacles will be formed, and persuasion which is out of time will cause a disease even a serious consequence, in addition, most college students still lack of related senses of psychological health[1-2]. However, application of psychological consultation gives college students psychological assistance and guidance and helps them develop their own potential and overcome psychological obstacles and mental and body diseases, being an effective method[3].

The traditional psychological consultation way basically is either to carry out a questionnaire survey or to have a face-to-face conversation, which has many disadvantages[4]. The purpose the paper develops a Web-based psychological consultation system is to solve clients' psychological problems by a virtual network, make them relax on psychology under loose network environment, improve measured realness and accuracy, and then manage and analyze their psychological consultation activities, reaching a goal which leads their psychology to develop at a healthy and reasonable direction, and make them really overcome themselves, face the life and pressure calmly, and cause the life more wonderful and comfortable[5].

\section{Requirement analysis of Web-based psychological consultation system}

Because users usually use e-mails now, so the system sets up the e-mail address of user as the login name, and the login password is not required to be identical with that of e-mail. The system just needs a right e-mail address in order to facilitate to correctly send users consultation results. At present, the number of people who use QQ is larger, and a younger generation basically has their own QQ number, therefore, the system allows to add the QQ consultation which adopts rich network communication functions of QQ software to greatly save the system burden ${ }^{[6]}$. The business flow chart of the system sees figure 1 . 


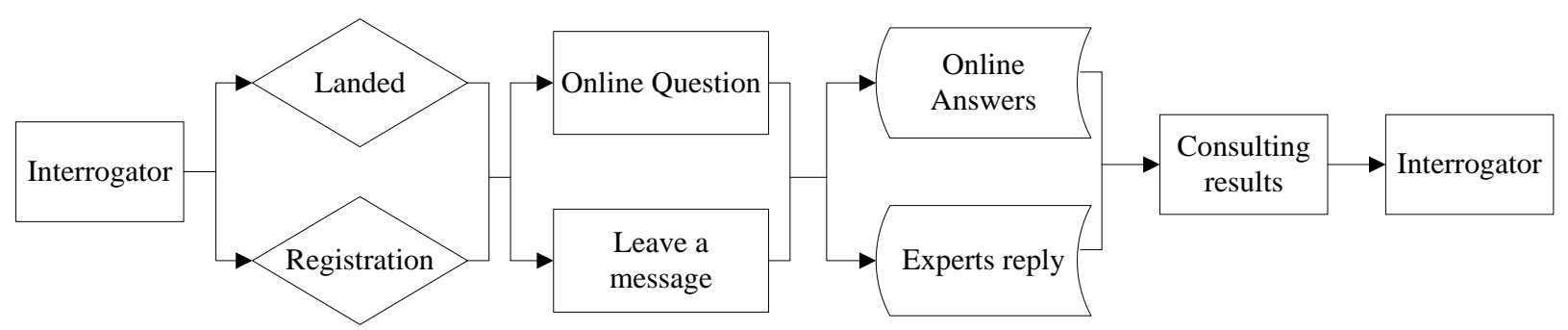

Fig.1 Business flow chart of psychological consultation

The system is required to meet the following functions:

Consultation management.

The consultation management refers that the psychological counseling expert communicates information with consultants by a network platform and a Q\&A system, thus automatically conducting an initial diagnosis. Consultation modes include two kinds: asynchronous consultation and synchronous consultation. The asynchronous consultation belongs to the most basic consultation way, and asynchronous working styles usually comprises: FAQ, e-mail consultation, form consultation, etc.. The system demands FAQ and form consultation, the two consultation ways, to be combined organically and makes them together service for counseling experts and consultants. In the question guidance, FAQ is added to reduce repeated questions which bring unnecessary workloads for experts and database maintainers. In form consultation, a way of e-mail is used to notify services of counseling expert and consultant, providing guarantee to solve problems within minimum time for consultants. The synchronous consultation is a real "face-to-face" Q\&A form, or real-time reference consultation. In the system, consultation services within short time are finished by text conversation, and the "face-to-face" consultation can be completed by QQ video function.

Information management.

The information management includes expert's information publicity and consultant's information protection.

User operation.

Users consist of: consultants and counseling experts. The consultant users are required to: register, login, question by guidance, find a password, etc.. And the expert users can enter, check and reply questions of consultant. Because psychological consultants do not hope personal information leak and psychological counseling experts have different computer levels, so the system performance requirements are: ensuring to keep personal information of consultant confidential; realizing a friendly interface: the system interface should be beautiful, simple and easy-to-use, facilitating consultants and counseling experts to do basic operation; applying real needs of psychological consultation and making the system have well extendibility, maintainability and adjustability.

\section{Structural design of system architecture}

Disposal procedures of system data. The system administrator of psychological consultation system is responsible for maintaining the system, including uploading some psychological knowledge and tests to the database and meanwhile deleting redundant files regularly from the database in order to reduce loads of database. Consultants and counseling experts save necessary information to the database by operation like register and message-leaving, and update relevant information in the database by correcting information.

Structural design of system function. According to basic procedures of online psychological consultation and maintenance requirements of system, system modules can be divided abstractly into user module, consultation module, database module, expert module and system maintenance and management module, which are shown as figure 2. 


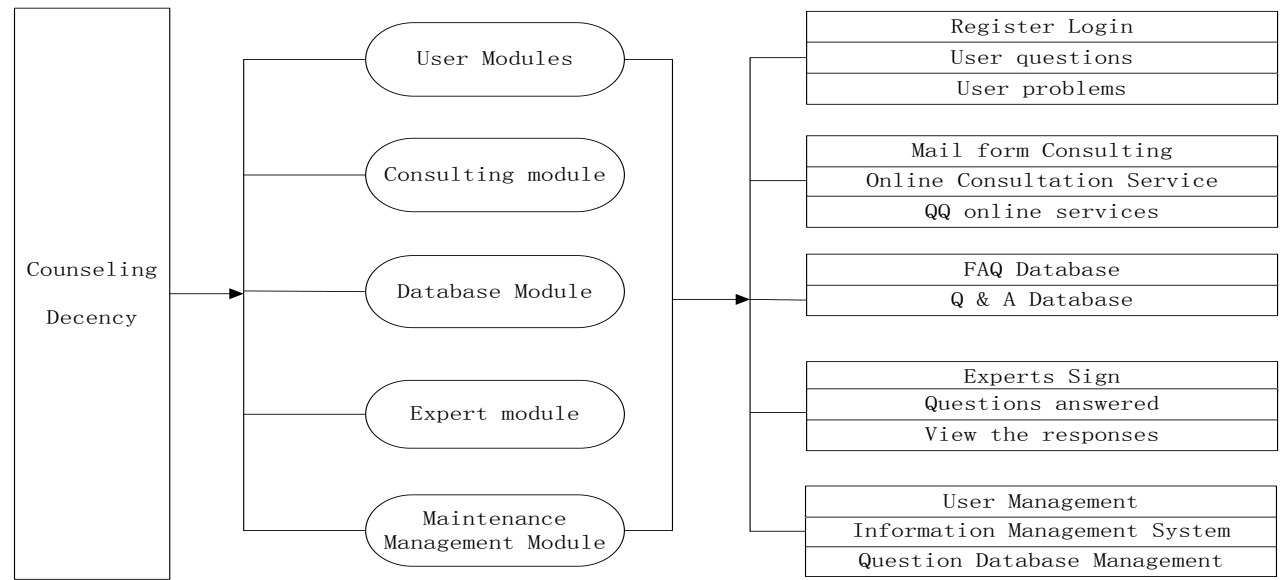

Fig. 2 Function structures of psychological consultation system

Design of system database. About 15,000 people use the system. The small-scale database (such as Access database) is competent if data records are little. However, with a gradual increase of data records, the small-scale database has its responding speed which obviously declines. In order to reduce problems of future database move, the system selects SQL Server 2005 as its backstage database. SQL Server is a representative of middle-scale database and a comprehensive database platform, which uses integrated business intelligence tools to offer enterprise database management. And SQL Server database engines are relation-based data and structured data. They offer more safe and reliable storage function and construct and manage high-performance data applications used in business. The E-R chart of system sees the figure 3 .

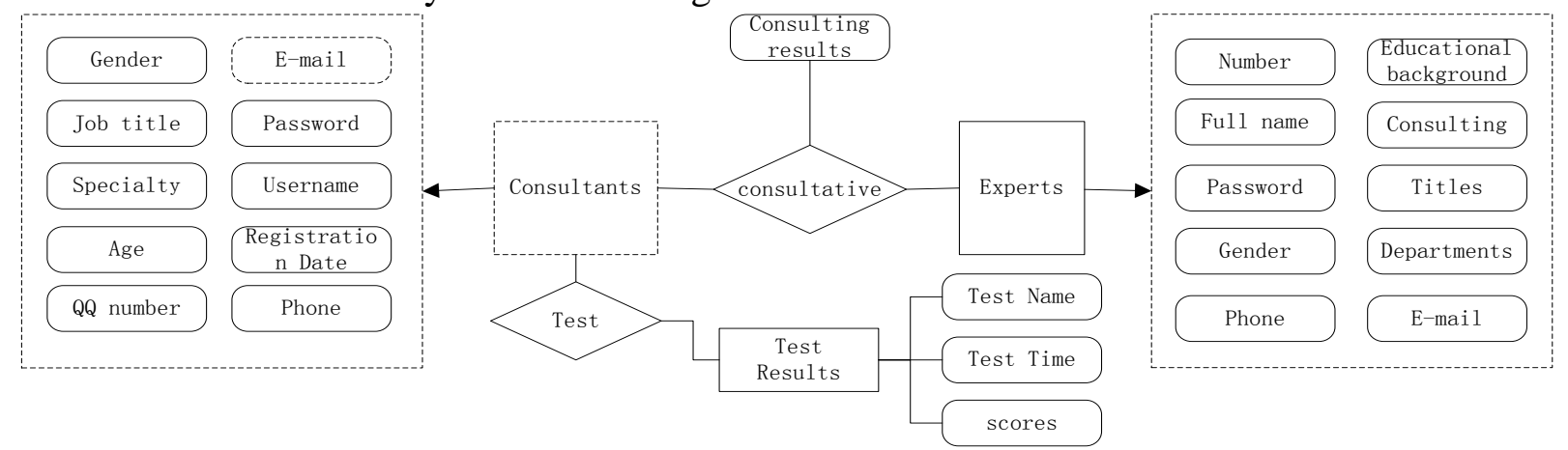

Fig.3 E-R chart of psychological consultation system

Design of consultation ways provided by system. The system provides various consultation ways for users to choose, including user consultation, expert consultation, real-time consultation and so on. The following will discuss operational processes of different consultation ways.

User consultation

The user module mainly includes user register, question submission, question check, check of question reply condition, customization of user information and collection of expert column. The user register mostly collects common information of user. The user may directly submit questions instead of registering, but offers an effective E-mail address for the sake of facilitating to receive answers automatically replied by system. The system regards the effective E-mail address submitted by user as the user login name, and then automatically generates a password which is sent to the user mailbox. The customization of user information is primarily to make users choose parts of services provided by system and regularly receive latest information issued by system. The collection of expert column means to chiefly collect a sheet of experts who are usually consulted by consultant himself/herself, facilitating to consult to the same expert again.

Expert consultation

The counseling expert enters the system unless first registers online and then passes examination. And experts must offer real materials. Main personal information includes name, sex, educational background, technical title, consultation field, telephone, E-mail, personal profile, etc.. Visitors can 
click the counseling expert list, check personal information of counseling expert, and choose a counseling expert according to personal characters of them. When counseling experts enter, they can see counseling questions which need to reply by themselves, and also answer public questions.when the counseling expert is replying questions, they will be classified into the database by system. And at the same time, answers will be sent to visitors in a form of e-mail. If the condition is enough, answers can be sent to visitor in a form of telephone message.

Real-time consultation

The design scheme of system adopts more practical online way--QQ plug-ins as well. The function of QQ software can meet basic requirements of consultation service. The biggest advantage of this software is instantaneity and reciprocity. As long as a user who uses it puts forward a question, he/she will receive an instant answer. The system utilizes the QQ plug-ins to put QQ numbers of counseling experts on the online counseling column in public. The plug-ins automatically displays an online state of counseling expert, and the user will directly send information to counseling experts as long as he/she just click a head icon. Through powerful communication function, the face-to-face remote assistant consultation is realized and thus reaches a purpose of consultation.

\section{Realization of Web-based psychological consultation system}

Whole planning of system website. According to website service contents and netizen requirements, combining the Internet features, and in the light of requirement plan, the system offers users to enjoy online service, and demands counseling experts to online reply various questions raised by users, accept related suggestions and proposals to the system by users and timely reply satisfactory answers to users online. And meanwhile, user's needs should be known according to messages left by users. In addition, the system also provides all-round consultation service in several forms such as online audio, video and words.

Realization of psychological knowledge browse function. The module chiefly targets are realizing a list of psychological knowledge and displaying of contents. For major functions, users can browse psychological knowledge in a form of list. If users know about psychological knowledge, they will see a list of psychological knowledge. Just a click of psychological knowledge title can enter to the content page, and then users may browse detailed contents.

Realization of system network forum function. The module mainly has functions like post publishing and reply, display of post list and display of post content. Major functions assist users to browse posts published in the online forum in a form of list. On the forum home page, it can be seen that the forum is made up of several parts, and each part has a name and some brief introduction. Then, the number of subject issued in each part and the number of subject reply will be counted. For major functions, users can publish a new post or reply the published post. In a page with a post published, when a user inputs the title and content of a post, the "save" button is just to be clicked then. If the post is saved successfully, a notice message which stands for successful save will be presented, and if not due to other reasons, a relevant notice message will also appear to hint to save again; if the user wants to input again, a "delete" button can be clicked; if the user wants to exit in the post publishing, an "exit" button can be selected. In the post reply page, detailed information of post will be firstly seen, for example, the publisher, publishing time, subject and content. If users desire to reply the post, they can input contents they want to reply in the reply textbox which is used for replying posts, and then just click the "reply" button. If the post is saved successfully, a notice message which stands for successful save will be presented, and if not due to other reasons, a relevant notice message will also appear to hint to save again; if the user wants to input again, a "delete" button can be clicked.

Realization of system user management function. The user management system mainly includes functions like user register, user login, personal management and user log-off. Its major functions are verification to user's identity, prevention to illegal user login and guarantee of system security. If the user identity is illegal, a relevant notice will appear; if not, links of "log-off" and "personal management" will appear. 


\section{Conclusion}

On the basis of research of related concepts and theory foundations on psychological consultation and relevant technical bases of system development, the paper adopts the B/S architecture, and uses ASP.NET programming technology and SQL Server database technology to develop a web-based psychological consultation system. After tests and practical use, it can be proved that the system is characterized by good convenience of accessing, strong real-time performance of searching, large scale of knowledge base, high professional degree of consultation service, etc. The system effectively offers psychological assistance and guidance to college students, help them develop their own potential, overcome psychological obstacles and mental and body diseases and face the life and pressure calmly in order to make the life more wonderful and comfortable.

\section{Acknowledgement}

In this paper, the research was sponsored by the Humanities and social science research project of heilongjiang province department of education (Project No.1252XS327)

\section{References}

[1] Wenbo Liang. College Students' Psychological Health Education Present Situation and Education Measures[J]. Theory Research.2011,(10):272-273.

[2] Wuxia Yang. Investigation and analysis of the factors affecting the mental health of college students[J]. Education Teaching Forum.2013,(47):108-109.

[3] Xiao Xiao. The Theory of College Students Psychological Counselling[J]. Journal of Hubei Institute of Education.2007,24(7):75-77.

[4] Ping Li. Practice \& Reflection of Psychological-Consultation Network of University Students[J]. Theory and Practice of Education.2009,29(11):38-40.

[5] Xiaocheng Chen. The Survey of the Current Status of Online Psychological Counsultants and Their Cognition of Online Psychological Counseling[J]. Health Medicine Research and Practice.2011.(1):55-57.

[6] Min Ouyang. Design of Psychological Consultation System in University Based on Web[J]. Science Mosaic.2010,(11):76-78. 